\title{
Teaching Approach of Emergency Traumatic Chest Injuries: A Review
}

\author{
Adel Hamed Elbaih MD ${ }^{1 *}$, Khaled Adnan Arnous ${ }^{2}$ \\ ${ }^{1}$ Associate Professor of Emergency Medicine, Faculty of Medicine, Suez Canal University, Ismailia, Egypt and \\ Associate Professor of Emergency Medicine, Sulaiman AlRajhi University, Clinical Medical Science, Saudi \\ Arabia \\ ${ }^{2}$ Emergency Medicine, College of medicine, Sulaiman AlRajhi University, Clinical Medical Science, Saudi \\ Arabia
}

*Corresponding Author: Dr. Adel Hamed Elbaih, Associate Professor of Emergency Medicine, Faculty of Medicine, Suez Canal University, Ismailia, Egypt.

\begin{abstract}
Background: Thoracic injuries are the third most common injuries in trauma patients, after injuries to head and extremities. Thoracic trauma has an overall mortality rate of 15-25\%, which is the highest with cardiac or tracheobronchial-esophageal injuries patients. The incidence of tension pneumothorax (TPT) varies with the population studied and is not well established.TPT would also seem to be more serious in ventilated patients reaching $91 \%$ mortality rates. Therefore, we aim to look into the common pitfalls that both medical students and new physicians face in the recognition, diagnosis, and chest injuries management.
\end{abstract}

Targeted Population: Chest injuries patients who are requiring urgent management in the ED, with Emergency Physicians for teaching approach protocol.

Aim of the Study: Appropriate for assessment and priorities for chest injuries patients by training protocol to Emergency Physicians. Based on patients' causes and types of chest injuries.

Conclusion: Chest Injuries are major problems should be corrected as they are identified. Do not delay treatment to obtain radiologic confirmation. Tube thoracostomy is mandatory after needle or finger decompression of the chest with continual reassessment of the patient is necessary.

Keywords: Chest Injuries, Emergency physicians, skill approach, management.

\section{INTRODUCTION (EPIDEMIOLOGY)}

Traumatic injuries account for about $37 \%$ of emergency department (ED) visits. Among all traumatic injuries, Thoracic injuries are the third injuries in trauma patients, after injuries to head and extremities. Thoracic trauma has an overall mortality rate of $15-25 \%$, which is the highest with cardiac or tracheobronchial-oesophageal injuries patients.

The incidence of tension pneumothorax (TPT) varies with the population studied and is not well established. often reflecting disease suspicion rather than true incidence.

In ventilated patients TPT is more likely if simple pneumothorax diagnosis is delayed. TPT would also seem to be more serious in ventilated patients reaching $91 \%$ mortality rates in one series. Most life-threatening thoracic injuries can be treated with airway controlor decompression of the chest with a needle, finger, or tube.

\section{DEFINITIONS, INDICATIONS CONTRAINDICATIONS}

TPT may be said to occur when a one-way valve communicates with the pleural space. Air is forced into the plural space with no way to escape, eventually collapsing the affected lung. Ultimately, pushesthe mediastinum to the opposite side, decreasing venous return and comprising the opposite lung (Figure 41).Tension pneumothorax is considered a lifethreatening emergency condition and requires rapid recognition and treatment. 


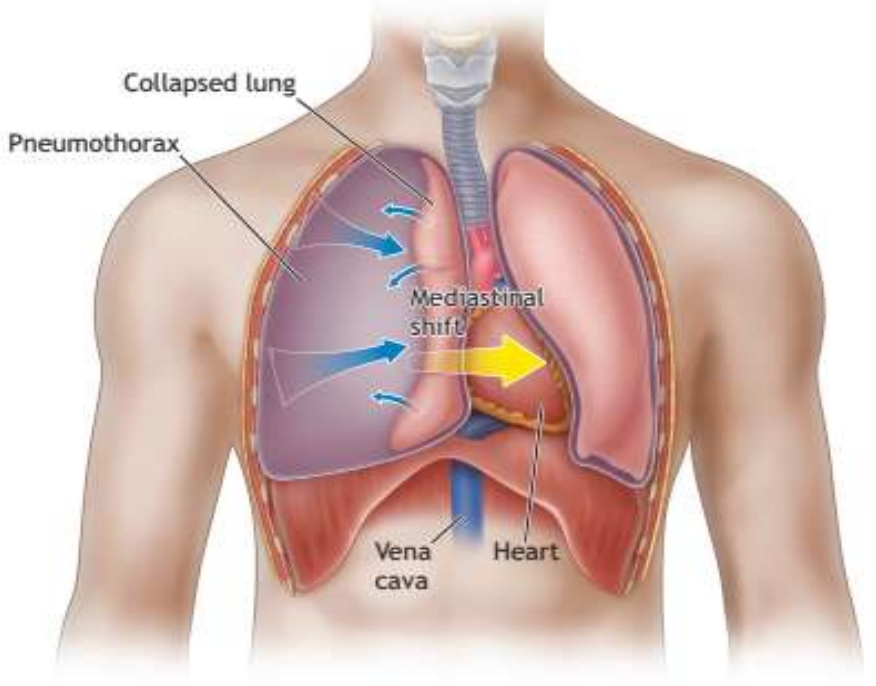

- FIGURE 4-1 Tension Pneumothorax. A "one-way valve" air leak occurs from the lung or through the chest wall, and air is forced into the thoracic cavity. eventually collapsing the affected lung.

Figure 4-1. Tension pneumothorax. A "One-way valve" air leak occurs from the lung or through the chest wall, and air is forced into the thoracic cavity, eventually collapsing the affected lung

The most common cause of tension pneumothoraxis mechanical positive-pressure ventilation in patientswith visceral pleural injury. But it is seen frequently following penetrating or blunt chest trauma in which parenchymal lung tissue fails to seal. Also, it could happen iatrogenically after attempted insertion of a central venousline. Tension pneumothorax ischaracterized by some or all of the following signsand symptoms (Figure 6.3).

The diagnosis of TPT is entirely clinical do not waste time waiting the radiological confirmation.

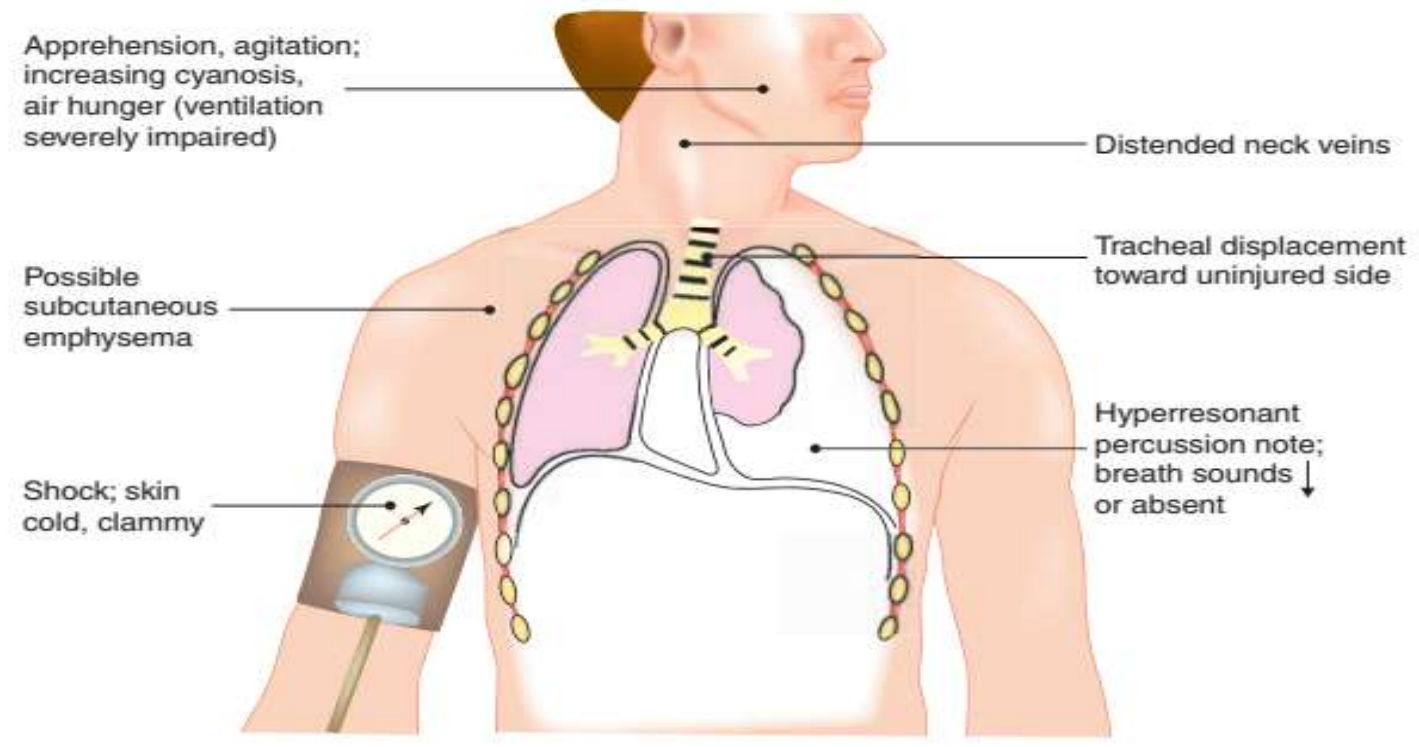

Figure 6.3

Tension pneumothorax. Campbell, John E., Basic Trauma Life Support for Advanced Providers, 5th ed., Copyright 2004. Reprinted by permission of Pearson Education, Inc., Upper Saddle River, NJ.

Figure 6.3. Tension pneumothorax. Campbell, John E., Basic Trauma Life Support for Advanced Provider, $5^{\text {th }}$ ed., Copyright 2004. Reprinted by permission of Pearson Education, Inc., Upper Saddle River, NJ.

3. Description of a Problem, a LACK of KNOWLEDGE ON A CERTAIN TOPIC OR A SEGMENT ON WHY THIS IS A PROBLEM

Tension pneumothorax (TPT) is an uncommon disease with a malignant course leading to death if untreated. It is most commonly encountered in prehospital trauma care, emergency departments, and intensive care units (ICUs). 
Resuscitation and trauma courses usually illustrate a patient in extremis and assume that the clinical diagnosis is straightforward and the response to needle chest decompression is rapid and reliable. However, this might not be the case in real life. Texts differ when describing the diagnostic symptoms and signs and there are several case reports of diagnostic difficulty or missed diagnosis because of an absence of "classic" signs. Lack of chest signs along with poor correlation between the signs present and those picked up by experienced physicians have been specifically noted. There have also been multiple reports of ineffective needle decompression with adequate treatment only being achieved with tube thoracostomy.

\subsection{WHY this Study is Necessary?}

The goal of studying and addressing these problems is to improve healthcare outcomes, decreasing the mortality rate, and highlight some of the wrong approaches applied by a physician in emergency cases. In addition to that, is to provide a standardized care for patient with tension pneumothorax. Any delay or missing diagnosis of tension pneumothorax will result in loss of live of the patient. Therefore, it is very important to apply the right approach of ATLS when dealing with such patient who present with sign and symptoms of TPT.

\subsection{Aim of Study}

This review study examines the present understanding of tension pneumothorax and produces recommendations for improving the diagnostic and treatment decision process.

\subsection{Study Question}

What is the mortality rate of patient present to ER department with TPT and how can we apply the ATLS approach to that patient?

4. DESCRIBE STEPS OF THE RIGHT Technique of This Method PoInt BY POINT

\subsection{Initial Management of Tension Pneumothorax}

As in all trauma patients, the primary survey of patients with thoracic injuries begins with the airway, followed by breathing and then circulation. Major problems should be corrected as they are identified. Diagnosis of tension pneumothorax is made clinically, before the chest $\mathrm{x}$-ray is obtained. Although the classic presentation includes distended neck veins, hypotension or evidence of hypoperfusion, diminished or absent breath sounds on the affected side, and tracheal deviation to the contralateral side (Figure 261-2), one or more of these elements may be absent in the presence of hypovolemia. Perform immediate needle decompression.

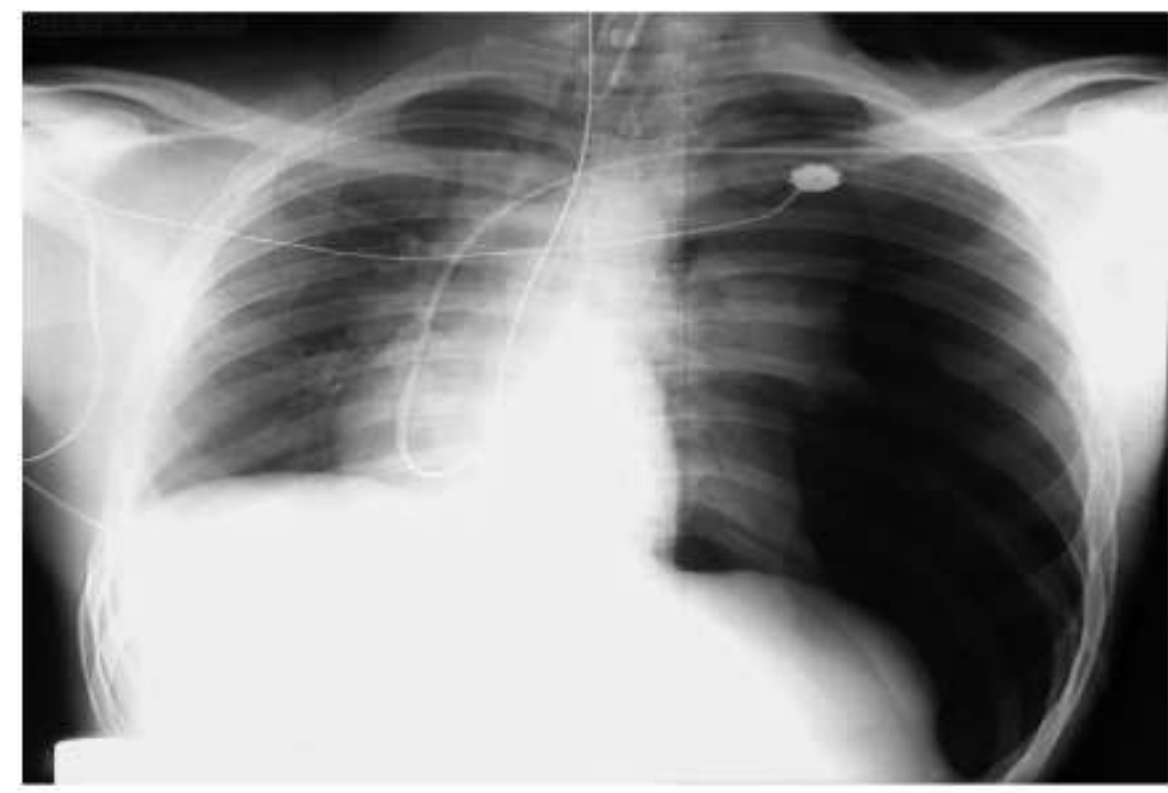

FIGURE $261-2$. Tension pneumothorax, Supine chest radiograph of a tension pneumothorax. Note the deviation of the trachea and shifting of the mediastinat contents to the right. Tension pneumothorax should normally be diagnosed prior to chest radiograph. IReproduced with permission from Schwartz DT (ed): Emergency Radiology, Case Studies. 2008 McGraw-Hill inc. Fig 1-9-10.] 
Figure 261-2. Tension pneumothorax. Supine chest radiograph of a tension pneumothorax. Note the deviation of the trachea and shifting of the mediastinal contents to the right. Tension pneumothorax should normally be diagnosed prior to chest radiograph. [Reproduced with permission from Schwartz DT (ed): Emergency Radiology, Case Studies. (C) 2008 McGraw-Hill Inc., Fig1-9-10.]

\section{Airway:}

Recognize and assess any major injuries affecting the patency and protection of the airway.

\section{Breathing:}

First of all, you should expose the patient's chest and neck completely to allow for assessment of neck veins and breathing. Assess the movement of the chest wall by looking at it and see if it's equal on both sides.

Listen to the chest to evaluate for equal breath sounds and identify any extra sounds that may indicate effusion orcontusion. Palpate to determine if there are areas of tenderness, crepitus, or defects.

Remember that cyanosis is a late sign of hypoxia in trauma patients and can be difficult to perceive in darkly pigmented skin; its absence does not necessarily indicate adequate tissue oxygenation or an adequate airway.

In case of TPT a hype-resonant breath sound will be noted on percussion, deviated trachea, and absent or diminished breath sound are all signs of tension pneumothorax. Arterial saturation should be assessed using a pulse oximeter and will be decreased when tension pneumothorax is present.

Tension pneumothorax requires immediate decompression and may be managed initially by rapidly inserting a large over-the-needle catheter into the pleural space. The most common approach to needle decompression is to introduce a 14-gauge IV needle and catheter into the pleural space in themidclavicular line just above the rib at the second intercostal space(see Figure 261-3).

The importance of anterior midclavicular approach lies because this is the shortest distance from the skin to the pleura,avoids theinternal mammary vessels that are locatedapproximately $3 \mathrm{~cm}$ lateral to thesternal border, and avoids mediastinal vessels. due to the variable thickness of the chest wall, needle decompression may fail in such cases. In this situation, finger thoracostomy is an alternative approach (see figure 4-2)

\section{TABLE 254-3 Primary and Secondary Survey in Trauma Resuscitation}

Primary Survey (rapid identification and management of immediately lifethreatening injuries)

A. Airway and cervical spine

Assess, clear, and protect ainway: jaw thrust/chin lift, suctioning.

Perform endotracheal intubation with in-line stabilization for patient with depressed level of consciousness or inability to protect airway.

Create surgical airway if there is significant bleeding or obstruction or laryngoscopy cannot be performed.

\section{B. Breathing}

Ventilate with $100 \%$ oxygen; monitor oxygen saturation.

Auscultate for breath sounds.

Inspect thorax and neck for deviated trachea, open chest wounds, abnormal chest wall motion, and crepitus at neck or chest.

Consider immediate needle thoracostomy for suspected tension pneumothorax.

Consider tube thoracostomy for suspected hemopneumothorax.

\section{Circulation}

Assess for blood volume status: skin color, capillary refill, radial/femoral/carotid pulse, and blood pressure.

Place two large-bore peripheral IV catheters.

Begin rapid infusion of warm crystalloid solution, if indicated.

Apply direct pressure to sites of brisk external bleeding.

Consider central venous or interosseous access if peripheral sites are unavailable.

Consider pericardiocentesis for suspected pericardial tamponade.

Consider left lateral decubitus position in late-trimester pregnancy.

\section{Disability}

Perform screening neurologic and mental status examination, assessing:

Pupil size and reactivity

Limb strength and movement, grip strength

Orientation, Glasgow coma scale score

Consider measurement of capillary blood glucose level in patients with altered mental status.

\section{E. Exposure}

Completely disrobe the patient, and inspect for burns and toxic exposures.

Logroll patient, maintaining neutral position and in-line neck stabilization, to inspect and palpate thoracic spine, flank, back, and buttocks.

Secondary Survey (head-to-toe examination for rapid identification and control of injuries or potential instability)

Identify and control scalp wound bleeding with direct pressure, sutures, or surgical dips. Identify facial instability and potential for airway instability.

Identify hemotympanum.

Identify epistaxis or septal hematoma; consider tamponade or airway control if bleeding is profuse.

Identify avulsed teeth or jaw instability.

Evaluate for abdominal distention and tenderness.

Identify penetrating chest, back, flank, or abdominal injuries.

Assess for pelvic stability; consider pelvic wrap or sling.

Inspect perineum for laceration or hematoma.

Inspect urethral meatus for blood.

Consider rectal examination for sphincter tone and gross blood.

Assess peripheral pulses for vascular compromise.

Identify extremity deformities, and immobilize open and closed fractures and dislocations. 


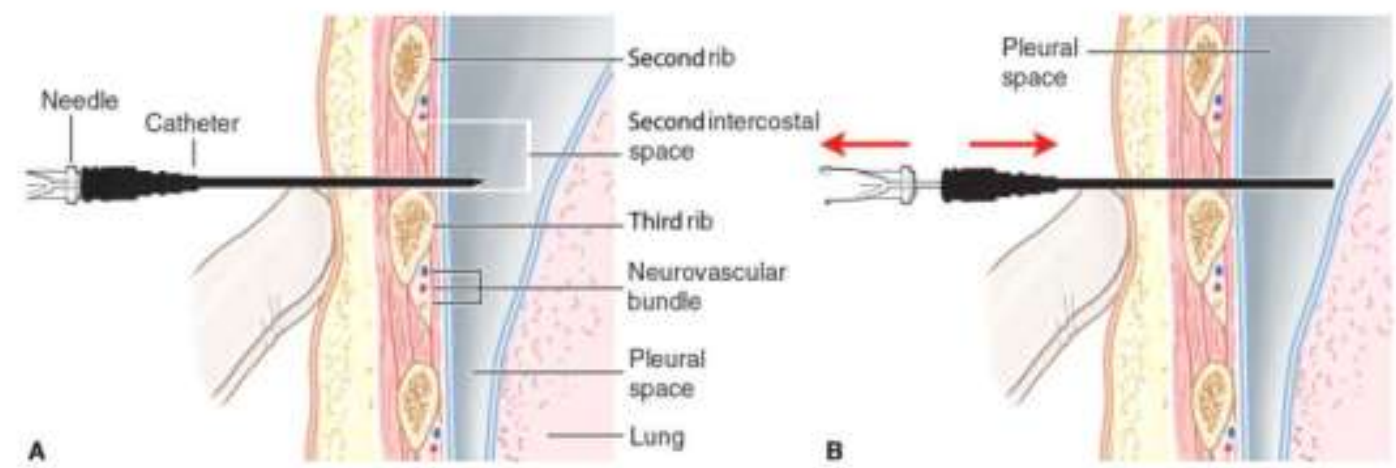

FIGURE 261-3. Decompressien of a tersion pneumothorar with a Gathestr-over-theneedle. A. The catheter-over-the-neede is inseted through the second intercostal space and into

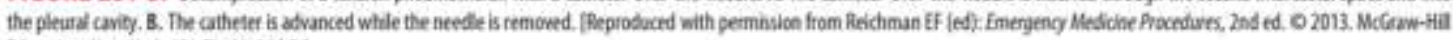
Education, New Yark, Nr. Fig 38-2As:

Figure 261-3. Decompression of a tension pneumothorax with a catheter-over-the-needle. $\boldsymbol{A}$. The catheter-overthe-needle is inserted through the second intercostals space and into the pleural cavity. B. The catheter is advanced while the needle is removed. [Reproduced with permission from Reichman EF (ed): Emergency Medicine Procedures, $2^{\text {nd }}$ ed. @ 2013. McGraw-Hill Education, New York, NY. Fig 38-2A\&B.]

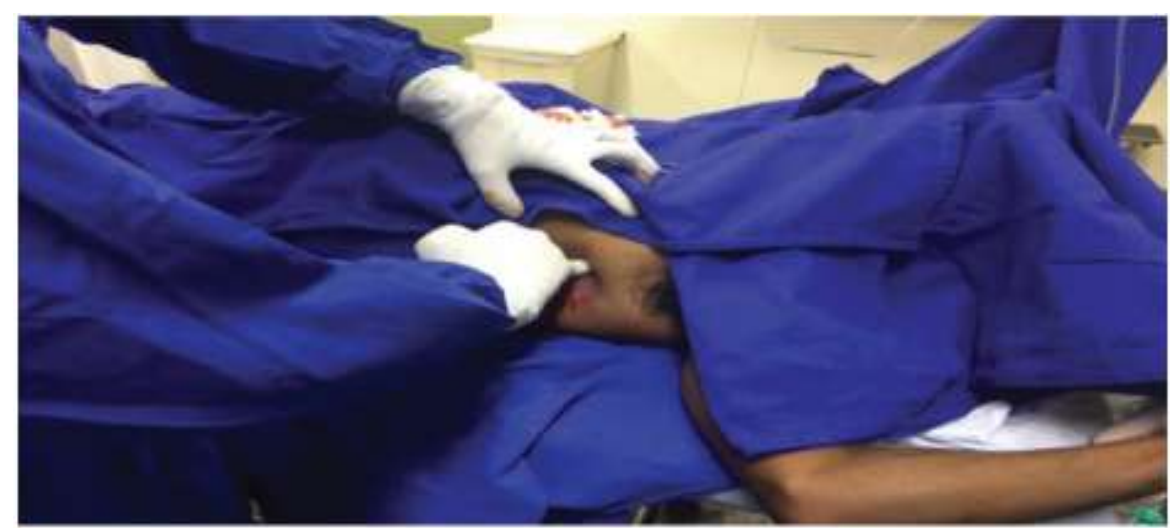

FIGURE 4-2 Finger Decompression. Tension pneumothorax can be managed initially by rapidly applying the finger decompression technique.

Figure 4-2. Finger Decompresson. Tension pneumothorax can be managed initially by rapidly applying the finger decompression

After a successful needle decompression,the tension pneumothorax is converted to a simple type. But there is a chance for recurrent pneumothorax due to the manoeuvre. So, reassessment of the patient and continuous monitoring is necessary. Tube thoracostomy is mandatory after needle or finger decompression of the chest as a definitive treatment.

It is very important during evaluation of breathing to differentiate between TPT and massive haemothorax (see table 4-1).

\begin{tabular}{|c|c|c|c|c|c|}
\hline \multirow[b]{2}{*}{ CONDITION } & \multicolumn{5}{|c|}{ PHYSICAL SIGNS } \\
\hline & $\begin{array}{l}\text { BREATH } \\
\text { SOUNDS }\end{array}$ & PERCUSSION & $\begin{array}{l}\text { TRACHEAL } \\
\text { POSITION }\end{array}$ & NECK VEINS & $\begin{array}{c}\text { CHEST } \\
\text { MOVEMENT }\end{array}$ \\
\hline $\begin{array}{l}\text { Tension } \\
\text { pneumothorax }\end{array}$ & $\begin{array}{l}\text { Decreased or } \\
\text { absent }\end{array}$ & Hyperresonant & Deviated away & Distended & $\begin{array}{l}\text { Expanded } \\
\text { immobile }\end{array}$ \\
\hline $\begin{array}{l}\text { Massive } \\
\text { hemothorax }\end{array}$ & Decreased & Dull & Midline & Collapsed & Mobile \\
\hline
\end{tabular}


Circulation: In tension pneumothorax patient may present with pulses electrical activity that manifested by ECG shows rhythm while the patient has no identifiable pulse. Figure 4-7 represents the algorithm for management of patient who present to ER department with circulatory arrest.

Skin should be inspected for mottling, cyanosis, and pallor.Neck veins should be assessed for distention, although in patient with tension pneumothorax and commitment hypovolemia they may not be distended. Listen for the regularity and quality ofthe heartbeat. central pulse should be assessed for quality, rate, and regularity.Palpate the skin to assess its temperature and determinewhether it is dry or sweaty.

Serial measurement of blood pressure and pulse oximetry should be done in patient with TPT.

* In trauma patient the stop point of fluid resuscitation is when all the following parameters: heart rate, blood pressure, distal pulses and capillary refill, urine output, mental status return to the normal value.

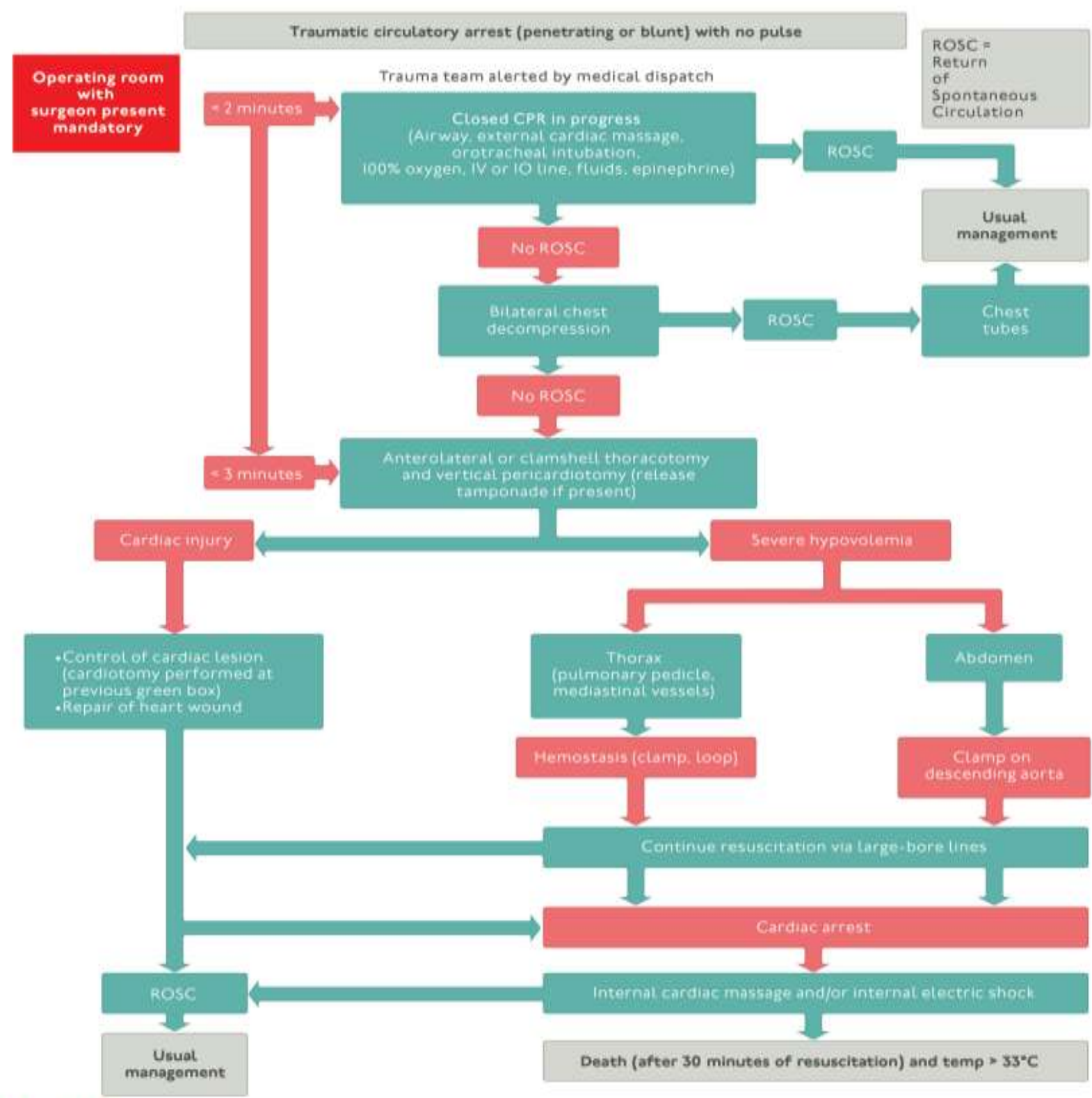

E FIGURE 4-7 Algorithm for management of traumatic circulatory arrest. ECM $=$ external cardiac massage; OTI $=$ orotracheal intubation: $\mathrm{IVL}=$ intravenous line; $1 \mathrm{OL}=$ intraosseous line.

Figure 4-7. Algorithm for management of traumaticcirculatory arrest. ECM=externel cardiac message; $O T I=$ orotracheal intubation; $I V L=$ intravenous line; $I O L=$ intraosseous line. 


\section{Disability:}

In tension pneumothorax patient, assessment of disability should be brief and specific to three major domains: level of consciousness, pupillary examination, and movement of extremities.

\section{Exposure and Environmental Control:}

Fully undress the patient from head to toe to allow a complete assessment. Failure to completely expose the patient may result in missing a significant traumatic injury, such as a gunshot or stab wound.

\subsection{Secondary Survey}

After treating and evaluating tension pneumothorax. patient should be involved in further serial chest X-ray, in depth physical examination, ongoing ECG, and pulse oximetry monitoring.

Checklist for acute management of tension pneumothorax

\begin{tabular}{l|l|l|l|l|}
\hline \multicolumn{5}{|c|}{ acute management checklist for tension pneumothorx } \\
\hline & Patient Management & Good & Fair & Poor \\
\hline 1 & $\begin{array}{l}\text { Directs assessment of airway, breathing, circulation, disability, and } \\
\text { exposure. }\end{array}$ & & \\
\hline 2 & Obtain vital signs including BP, HR, TEMP, RR, mental status & & & \\
\hline 3 & Administer high-concentration supplemental oxygen (100\% FiO2). & & & \\
\hline 4 & Avoid positive pressure ventilation. & & & \\
\hline 5 & $\begin{array}{l}\text { Perform emergency needle decompression if the patient is hemodynamically } \\
\text { unstable. followed by tube thoracostomv. }\end{array}$ & & & \\
\hline 6 & serial X-ray. & & & \\
\hline 7 & Continuous telemetry, continuous pulse oximetry & & & \\
\hline 8 & Transfer to ICU. & & & \\
\hline 9 & Case Conclusion & & & \\
\hline
\end{tabular}

\section{REFERENCES}

[1] Tintinalli, Judith E.Cline, David,eds. Tintinalli's Emergency Medicine Manual. New York : McGraw-Hill - Medical, 2012. Print.

[2] CVA, K., 2017 https://www.medwinpublishers.com/JOBD/JO BD16000139.pdf. Journal of Orthopedics \& Bone Disorders, 1(7).

[3] Mahadevan, S. and Garmel, G., 2011. An Introduction To Clinical Emergency Medicine. Cambridge: Cambridge University Press.

[4] Elbaih, A., 2017. Patterns and management of chest injuries patients and its outcome in Emergency Department in Suez Canal University Hospital, Egypt. Medicine Science | International Medical Journal, p.1.

[5] Flynn, J., Choi, M. and Wooster, L., 2013. Oxford American Handbook Of Clinical Medicine. Oxford: Oxford University Press.
[6] Roberts DJ, Leigh-Smith S, Faris PD, et al. Clinical manifestations of tension pneumothorax: protocol for a systematic review and meta-analysis. Syst Rev. 2014;3:3. Published 2014 Jan 4. doi:10.1186/2046-40533-3.

[7] Zarogoulidis P, Kioumis I, Pitsiou G, et al. Pneumothorax: from definition to diagnosis and treatment. J Thorac Dis. 2014;6(Suppl 4):S372-S376. doi:10.3978/j.issn.20721439.2014.09.24.

[8] Leigh-Smith S, Harris T. Tension pneumothorax--time for a re-think?. Emerg Med J. 2005;22(1):8-16. doi:10.1136/emj.2003.010421

[9] Renouf T, Parsons M, Francis L, et al. Emergency Management of Tension Pneumothorax for Health Professionals on Remote Cat Island Bahamas. Cureus. 2017;9(6):e1390. Published 2017 Jun 25. doi:10.7759/cureus.1390. 
[10] Gaillard, F., 2020. Tension Pneumothorax | Radiology Reference Article | Radiopaedia.Org. [online] Radiopaedia.org. Available at: $<$ https://radiopaedia.org/articles/tensionpneumothorax $>$ [Accessed 8 June 2020].
[11] Yoon, J.S., Choi, S.Y., Suh, J.H. et al. Tension pneumothorax, is it a really life-threatening condition?. J Cardiothorac Surg 8, 197 (2013). https://doi.org/10.1186/1749-8090-8-197.

Citation: Adel Hamed Elbaih MD, Khaled Adnan Arnous. Teaching Approach of Emergency Traumatic Chest Injuries: A Review. ARC Journal of Surgery. 2020; 6(2):1-8. DOI: https://doi.org/ 10.20431/2455$572 X .0602001$.

Copyright: (C) 2020 Authors. This is an open-access article distributed under the terms of the Creative Commons Attribution License, which permits unrestricted use, distribution, and reproduction in any medium, provided the original author and source are credited. 